\title{
Cellular Automata Simulation of Urban Traffic Flow Considering Bus Lane
}

\author{
http://dx.doi.org/10.3991/ijoe.v9iS7.3193 \\ Tianqi Zhou ${ }^{1}$ and Lijuan Peng ${ }^{2}$ \\ ${ }^{1}$ Sichuan University, Chengdu, China \\ ${ }^{2}$ Southwest University of Science and Technology, Mianyang, China
}

\begin{abstract}
Based on the analysis of urban traffic flow affected by bus lane, a two-lane cellular automata model is established under the periodic boundary condition. The properties of traffic flow are investigated by analyzing the velocity-density and flow-density diagrams. The spatial-temporal diagrams with the two types of bus stop facilities are also discussed. Simulated results show that the bus lane can significantly improve the traffic flow, and the volume can increased by extending the bus stop length, and the bus stop with no overtaking facilities has less volume than the bus stop to permit overtaking.
\end{abstract}

Index Terms-Mixed traffic flow, Bus lane, Cellular automata, Simulation

\section{INTRODUCTION}

The urban traffic flow affected by public transit system has aroused the concern of many scholars in recent years. Scholars have studied theories and models of public transit system from different angels and many models have been proposed, such as car-following model, hydrodynamic model, and cellular automata model. In particular, cellular automata models with their ability to simulate both gross and subtle features of real traffic have gotten comprehensive research and application [1-6]. X.M. Zhao, Z.Y. Gao and B. Jia $[7,8]$ studied the combined effects of the signalized intersection and its near-by bus stop using a two-lane CA model, and the effect of the bus stop between two neighboring intersection were investigated. Y.S. Qian, H.L. Wang and C.L. Wang [9] developed a CA model with different maximum speed vehicles mixed on single lane which considers the effect of harbor-shaped bus stop. B. Jia, X.G. Li and R. Jiang [10] investigated the influences of the non-harbor shaped bus stop and the harbor shaped bus stop on the traffic flow. A two-lane CA model is proposed by D.Q. Li , L.Y. Dong and S.Q. Dai [11] to simulate the traffic bottleneck induced by bus stopping. C.Chen, T.Wang and C.Y Li.et al [12] have studied the setting conditions of bus bay stop. A model of mixed vehicle with traffic parameters, such as bus-car volume, vehicle volume and ratio of bus-car and put forward running speed, is introduced by X.G. Yang, H. Xu and J. Wang[13]. Y.J. Liang and Y. Xue[14] studied the impact of the arrangement of bus stops without bus bay. A certain characteristics of the bus stop without stopping-lane were analyzed by S.J. Kang and Y. Xue[15]. However, as the important part of bus priority measures, little information has been published concerning the impacts of the bus lane, which is a bus track infrastructure where buses are separated from other traffic by road marking or studs, to the traffic flow.

Based on the NaSch model, a new two-lane cellular automata model considering the influence of the bus lane section on urban traffic flow under the periodic boundary condition is established. The model also considered the different driving rules and lane change rules between the car driver and the bus driver under the mutative traffic condition. For the section with bus lane and the section with off-line bus stops, the distribution of velocity-density and flow-density are provided. It is found that bus lane can significantly improve the traffic flow. By extending the length of the bus stop, the bus volume can be increased. And for the conditions studied, the provision of overtaking bays is likely to have a greater effect on speeds than does the provision of bus ordering.

\section{Cellular automaton tRafFiC Flow MODEL With BUS LANE}

The two-lane CA model is built from the two-lane road under the periodic boundary condition. It is assumed here that vehicles move only in one direction. The road is illustrated in Fig. 1 with two lanes, the right and the left, which each lane of the road is divided into $L$ cells. The road consists of two sections with same length. Section bus lane is the start of the road which the right lane is only use for buses. Section general traffic is the part between the middle of the road and the end of the road which the lane changing is permitted. The upstream part near the bus stop is section A and special lane-changing rules will be used in this section. There is one intersection between bus lane and general traffic section. For each section, there is one off-line bus stop with several bays (from the right to the left named Bay1, Bay2....) lies at the middle of the right lane (or the bus lane).

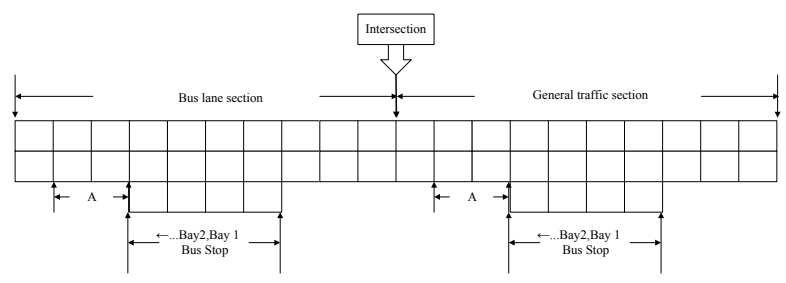

Figure. 1 The road configuration in the simulations.

There are two types of vehicles including car and bus considered in the simulations. Buses mix in the traffic flow with ratio $R . \rho$ denotes the density of the vehicles on the whole road. $L_{s}$ stands for the length of the bus 
stop. $T_{s}$ denotes the dwell time of stopping buses. The updating process of the traffic system includes two steps: forward motions and lane changing motions. And the whole updating for all vehicles is performed simultaneously.

\section{A. Forward motions}

The NS model is used to control the vehicles moving forward in this paper. The variable $v_{n}^{i}(t)$ denotes the speed of the vehicle $i$ on the lane $n$ at time $t$ which have to be integers between 0 and $v_{\max }$. The speed limit of the bus is denoted by $v_{\max }^{b}$, and the $v_{\max }^{c}$ is the speed limit of the car. $P$ is the probability to randomly decelerate. $X_{n}^{i}(t)$ is the position of vehicle $i$ on the lane $n$. At each discrete time step the system is updated by the following rules:

(1) Acceleration:

$$
V_{n}^{i}(t+1)=\min \left(V_{n}^{i}(t)+1, V_{\max }\right) .
$$

(2)Deceleration:

$$
V_{n}^{i}(t+1)=\min \left(V_{n}^{i}\left(t+\frac{2}{3}\right), \operatorname{gap}^{i}(t)\right) .
$$

(3)Randomization with probability $P$ :

$$
V_{n}^{i}\left(t+\frac{2}{3}\right)=\max \left(V_{n}^{i}\left(t+\frac{1}{3}\right)-1,0\right)
$$

(4) Motion:

$$
X_{n}^{i}(t+1)=X_{n}^{i}(t)+V_{n}^{i}(t+1)
$$

In the formulas above, $l e n_{n}^{i}$ is the length of vehicle $i$ on the lane $n$, and $g a p^{i}(t)$ is the gap between vehicle $i$ and its preceding vehicle $i+1$ at time $t$ which is calculates by (5).

$$
\operatorname{gap}^{i}(t)=X_{n}^{i+1}(t)-X_{n}^{i}(t)-l e n_{n}^{i}
$$

Define $D S_{n}^{i}(t)$ as the gap between the bus $i$ and the bus stop and $X_{\text {stop }}$ is the position of the bus stop entry.

$$
D S_{n}^{i}(t)=X_{\text {stop }}-X_{n}^{i}(t)-\operatorname{len}_{n}^{i}
$$

In cases with the stop lane or the bus lane when the buses near the bus stop,

$$
D S_{n}^{i}(t)<v_{\max }^{B}
$$

the deceleration rule of the buses will follow the added rules (8), it means bus $i$ will enter the bus stop with speed 1 as the first vehicle in the bus stop and move to the Bay 1 when the bus stop is empty.

$$
v_{n}^{i}(t+1)=\min \left(D S_{n}^{i}(t), v_{n}^{i}(t+1)\right)
$$

If the Bay 1 is allocated, the bus will enter the stop with speed 1 and move to the Bay 2 as the second vehicle in the bus stop, and so on. And if all of the bays of bus stop are already been allocated, all the stopping buses will queue at the entry to the stop with speed 0 .

When the stopping bus $i$ staying Bay $n$ at the bus stop, it can move forward with speed 1 if the bay $n-1$ is empty then its speed is updated as 0 . If the bus staying in the Bay 1, it cannot move forward if its dwelling time is less than $T_{s}$ which speed is updated as 0 . The bus becomes a non-stop bus when the dwelling time is equals $T_{s}$ and then waiting for exit the bus stop (for the general traffic section).

\section{B. Lane-Changing Motions}

Each of iteration consists of the two sequential steps. First the vehicles change lanes, then the position update follow the rules. For the bus lane which buses and cars are separated, there is no lane changing motion. When the vehicles pass through the intersection or the end of the road, it will enter the bus lane section. At this time, vehicles will change to the certain lane. And for simplicity, the lane-changing is finished in the intersection (or the end of the road) with no restrict.

There is different driving behavior between the car drivers and the bus drivers under the traffic condition. The drivers of buses are willing to run on the right lane to stop conveniently when they are close to the bus stop. However the drivers of cars are willing to run on the left lane to move quicker and avoid the block by the buses enter the bus stop or queue at the entry of stop. Therefore the lane-change probabilities of buses and cars are different from each other. Define $P_{c}$ as the probabilities of the vehicle, $P_{c}^{c a r}$ for cars lane-changing probability, and $P_{c}^{\text {bus }}$ for cars lane-changing probability, $0<P_{c}^{b u s}<P_{c}^{c a r}<1$.

In general traffic section vehicles will change lanes as the following rules.

(1)If the road situation on the present lane is not much better than that on the other lane and there are enough space to guarantee the safety, vehicles change lane with its lane change probability $P_{c}^{b u s}$ or $P_{c}^{c a r}$.

$$
\begin{gathered}
g a p^{i}<\min \left(v_{n}^{i}(t), v_{\max }\right) \\
g a p_{n}^{i}>g a p^{i} \\
g a p_{n B a c k}^{i}>v_{\text {back }}^{\max }
\end{gathered}
$$


Define $\operatorname{gap}_{n}^{i}$ as the gap between vehicle $i$ and its preceding vehicle on the destination lane. The variable $\operatorname{gap}_{n B a c k}^{i}$ in the above formulas means the gap between vehicle $i$ and its back vehicle on the destination lane.

(2)The drivers of car are willing to change lane when its preceding vehicle is bus. If the following condition is satisfied, the car will change lane with probability $P_{c}^{c a r}$.

$$
\begin{gathered}
\operatorname{gap}_{n B a c k}^{i}>1 \\
\operatorname{gap}_{n}^{i} \geq 0
\end{gathered}
$$

(3)All cars on the road and the stopping buses which do not lie in Section A will change lanes. The cars will change to the left lane and the bus will change from the left lane to the right lane as long as conditions on the right lane are safety.

$$
\begin{gathered}
\operatorname{gap}_{n}^{i}>=0 \\
\operatorname{gap}_{n B a c k}^{i}>=v_{n}^{i-1}(t)
\end{gathered}
$$

The same changing rules above are used for stopping buses which will change lane in order from bus stop to the right lane (or bus lane) when the dwell time is satisfied. If a stopping bus can not change to the destination lane, it will stop and wait for opportunity to change in order.

The periodic boundary condition is used in the simulations. In each time step, the position of the last vehicles on each lane is checked when the update of all vehicles is finished. If the position of the leading vehicle is beyond the length of the road, it will be move to the end of the queue on the certain lane as the lane-changing rule mentions above and its following vehicle becomes the new leader.

\section{RESULTS AND DISCUSSIONS}

The standard simulations set-up in this paper is described for the following observations. The road with length $7.5 \mathrm{~km}$ is divided into $L=2000$ cells. Bus lane section and general traffic section are all contain 1000cells. The Section A consists of 8 cells. The lengths of each cell correspond to $3.75 \mathrm{~m}$. The length of car is 2 cells and $v_{\max }^{c}=5 \mathrm{cells}=67.5 \mathrm{~km} / \mathrm{h}$. The length of bus is 4 cells and $v_{\max }^{b}=3$ cells $=40.5 \mathrm{~km} / \mathrm{h}$. The limits of the speed are reasonable values that match the actual situation of the urban expressway which with the high volume and low speed. Each time step corresponds to 1 second. The model parameters are set as follows: $P=0.1, P_{c a r}=0.3, P_{b u s}=0.2$. The flux is averaged over $1 \times 10^{4}$ time steps, and the first $5 \times 10^{3}$ time steps are discarded to avoid transient behavior.

At first, the influence of bus lane on the traffic flow as $L_{s}=8$ cells with different bus ratio is considered.
According to the Transit Co-operative Research Program Report 19(1996)[16], the average peak-period dwell time exceeds 30 second per bus, so $T_{s}=30 \mathrm{~s}$ in this section. One of the most basic measurements in traffic flow model is the fundamental diagram, which represents a relation between the three macroscopic variables: average velocity $v$, flow $q$ and density $\rho$.For the two types section, the relationship between cars flux and density with different $R$ is shown in Fig. 2. And the relationship between cars velocity and density is shown in Fig.3. Since buses and cars are separated running on the bus lane section, the cars flux and velocity only be affected by density but not be affected by the bus ratio.

From Fig. 1 and Fig.2, it can be seen that:

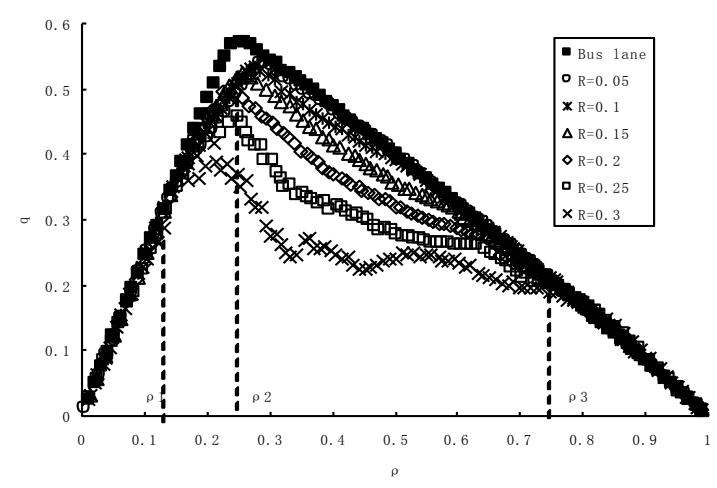

Figure. 2 The relationship between flux and density with different ratio of bus

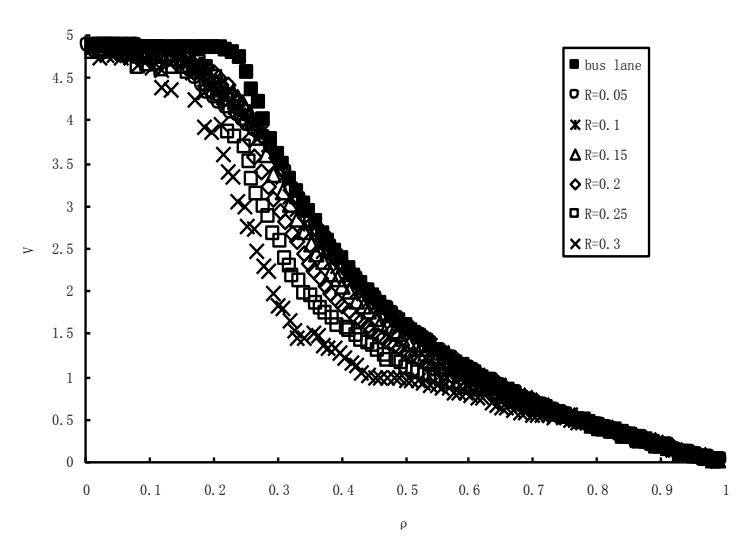

Figure. 3 The relationship between velocity and density with different ratio of bus

(1) When the density is low, $\rho<\rho_{1}$, the flux increases with the density which the traffic flow on the two section is free flow. In comparison with bus lane section, since $v_{\max }^{b}<v_{\max }^{c}$, cars may block by buses which the maximum speed are lower or in the circumstance which the buses change lane to enter the bus stop, the velocity of vehicles on general traffic section decreases as $R$ which is shown in Fig.3. The average velocity of the vehicles on the bus lane section is a little superior to the general traffic section. At this time, the effect of bus lane is not very significant. 
(2)When $\rho_{1}<\rho<\rho_{3}$, it is clear shown in Fig.2 that as $R$ increases, flux of cars on the general traffic section grows until it approaches a maximum value then drops with the density grows. For the ratio of bus increase, the number of bus is rising, so does the lane-change motions of the buses and the queue at the entry of bus stop. Therefore the maximum flux and velocity get smaller when the $R$ increase.

The flux of the bus lane section reaches its maximum value at density $\rho_{2}$, which is larger than all maximum flux value of general traffic section with different $R$.And Fig. 3 shows that, the average velocity of the vehicles on the bus lane section grows until it approaches a maximum value and then stay at the value as long time than it on the general traffic section. At this time, the flux and velocity of bus lane section are all higher than that of general traffic section.

(3) When $\rho>\rho_{3}$, the traffic flow is congested in both sections because of buses queue at the entry hinder the upstream vehicles from moving forwards. The bus stop has a bottleneck effect on both bus lane section and general traffic section. At this time, the flux of car can not be increased by create bus lane.

The above results indicate that bus lane can keep the traffic flow more freely especially the bus ratio $R$ keep a larger value. To further understand the effect of bus lane, the volume and velocity of buses with the different length of bus stop is considered when $R=0.15$.

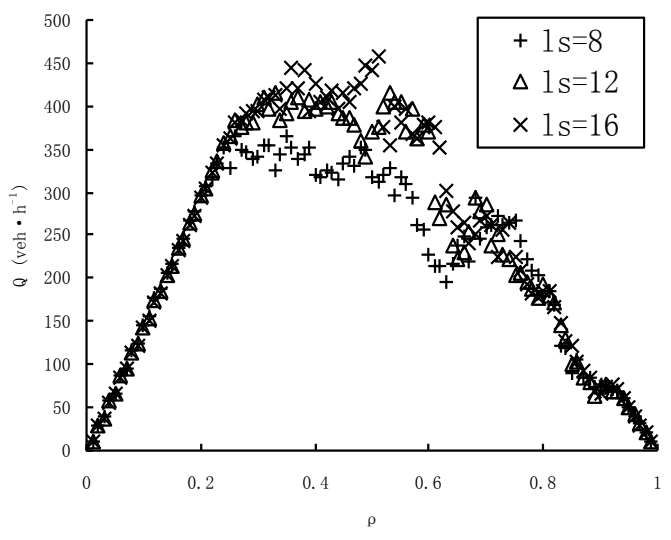

Figure. 4 The flux of bus on the general traffic section with different length of bus stop

From Fig.4, the flux of buses shows limited growth by extending the length of bus stop on the general traffic section.

When the traffic is very light ( $\rho<0.3$ ), the growth of flux on general traffic section is close to the flux on bus lane. At this stage, the maximum of the flow values approach $\mathrm{Q}_{1}=413 \mathrm{vel} / \mathrm{h}$ on the general traffic section and $\mathrm{Q}_{2}=404 \mathrm{vel} / \mathrm{h}$ on the bus lane section. The $Q_{2}$ is less than $Q_{1}$ can be explained that bus drivers can improve the velocity by lane-change motions on the general traffic section.
When the traffic is heavy ( $\rho>0.3$ ) in the Fig.4, the flux increase until it approaches a maximum value. For the different length of the bus stop, the maximum value of flux in a small range from $365 \mathrm{vel} / \mathrm{h}$ to $457 \mathrm{vel} / \mathrm{h}$. Then the flux of buses decline very greatly until 0 when $\rho>0.7$. At this time, the cars on the downstream of bus stop block the exit of buses from the bus stop, and then the dwell time becomes larger. The buses waiting outside the entry accumulate greatly at the same time. The flux of bus is affected seriously by the interaction between cars and buses.

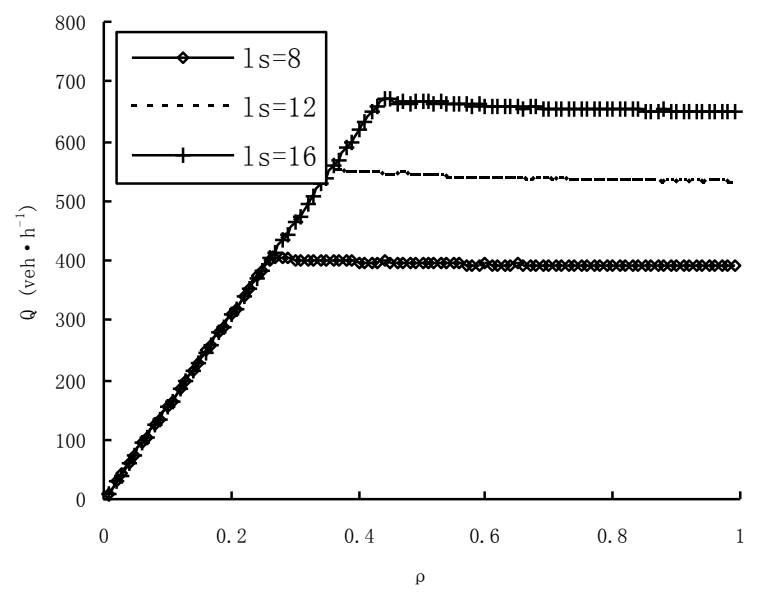

Figure. 5 The flux of bus on the bus lane section with different length of bus stop

In the Fig.5, the flux first grows until it approaches a maximum value which is increased with $L_{s}$, then remains at this value on the bus lane section. The maximum value of flux is increased effectively from $413 \mathrm{vel} / \mathrm{h}$ to $768 \mathrm{vel} / \mathrm{h}$. It is worth noting that the minimum value of the range $413 \mathrm{vel} / \mathrm{h}$ when $L_{s}=8 \mathrm{cell} s$ on the bus lane section is very close to the maximum value $457 \mathrm{vel} / \mathrm{h}$ which is reached by the section with 16 cells length bus stop. In addition, the flux is still very high even if the density is large.

It is found that, the length of bus stop has more influence on the bus lane section than on the general traffic section. And the flux of buses can be effective improved by increasing length of bus stop on the section which set a bus lane.

However the length of bus stop can not immeasurably increased in reality. In the above results, buses enter the off-line bus stop in order and there is no special facility in bus stop. In addition, with the different of predominant passenger movement, the buses dwell times are different between boarding predominates which services run overloaded or alighting predominates (or express services) which those services run nearly empty [17].

Considered two types of buses in the ratio $1: 1$, a short dwell time $T_{\text {alighting }}=30 \mathrm{~s}$ for alighting predominate buses and a long dwell time $T_{\text {boarding }}=60 \mathrm{~s}$ for boarding predominate buses. The time-space plot for the right lane on bus lane near the off-line bus stop with three bays 


\section{( $L_{s}=12$ ) when $R=0.15$ and $\rho=0.3$ from 9000-9500 is displayed in Fig.6.}

It can be seen a clustering phenomenon in the upstream section of the bus stop. In downstream section of the bus stop, there is no hindrance for the buses which waiting for stop to proceed. Thus, the queue at the entry of the bus stop is accumulate by the increase of buses dwelling time in the bus stop. However some of the buses finish their stopping procedure at the stop already cannot leave out of the bus stop because the first bus in the bus stop is not finish the procedure because of the buses entry and exit the bus stop must be in ordered.

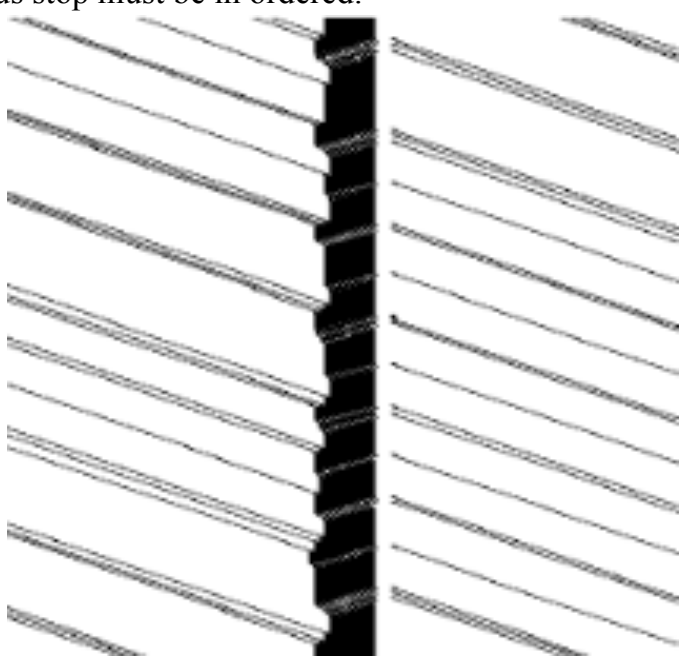

Figure. 6 The time-space plot of general off-line bus stop with 3 bays on the bus lane section

To improve the throughput of bus stop, a bus stop layout and operating regimes is introduced in this section which is familiar in the form of bus priority in many countries [17].

Fig.7 shows a 3-bay off-line bus stop with bus overtaking facilities in the bus stop area. It is different with the general off-line bus stop which all bays can be provided for all stop buses; there is a special bay $\mathrm{C}$ which can only be allocated to limited stop. The boarding predominate buses can only be allocated to bay A or Bay B. The alighting predominate buses may either be allocated to specific bay $\mathrm{C}$ or use any bay.

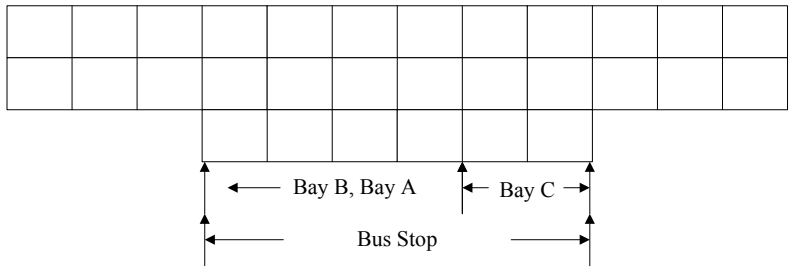

Figure. 7 Off-line bus stop with bay to permit overtaking on the bus lane section

Change the position update rules for the stopping buses as follow.

(1)For the boarding predominate bus, it will enter the bus stop in order, and move from Bay B to Bay A if Bay $\mathrm{A}$ is empty in its dwelling time. If Bay A and Bay B are occupied by other buses, it will queue to access the stop. All of the boarding predominate buses will stay at Bay A to waiting for exit the bus stop until the dwelling time and safe condition are satisfied.
(2)For the alighting predominate bus, it will be allocated preferentially to Bay $\mathrm{C}$ if the Bay $\mathrm{C}$ is empty, no matter the Bay A and B empty or not. And then exit the bus stop from Bay C. Else if Bay $\mathrm{C}$ has been occupied, the bus will enter the Bay A or Bay B if any of them is empty. Then it will change lane from bus stop to the right lane as well as the dwell time and safety conditions are all satisfied.

Fig. 8 shows the time-space diagram for the bus stop with overtaking facilities when $R=0.15$ and $\rho=0.3$ from 9000 time steps to 9500 time steps. From the Fig. 8 , the simulation indicates that the queue at the entry of bus stop becomes shorter and fades out quicker than that of in the Fig.6. This suggests that for the conditions studied, the provision of overtaking bays is likely to reduce the delays of the buses in the stop.

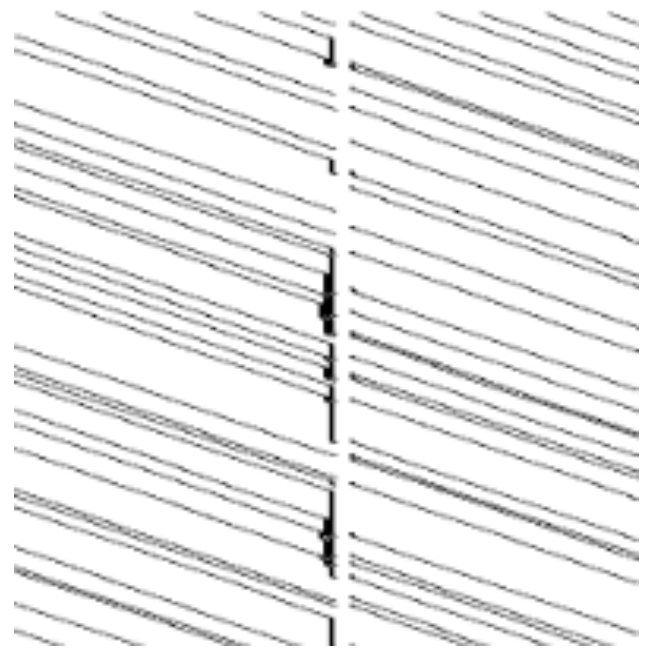

Figure. 8 The time-space plot of off-line bus stop with special Bay C to permit overtaking

The flux and density diagram for two types of off-line bus stop mentions above is shown in Fig.9. It can achieve relatively high volume for the overtaking bus stop which maximum value is reached $618 \mathrm{veh} / \mathrm{h}$ as $\rho=0.44$. This value is superior to the general off-line bus stop which the maximum value is $550 \mathrm{veh} / \mathrm{h}$ as $\rho=0.36$. And the flux is close to the general off-line bus stop with 4 bays which the maximum value is $669 \mathrm{veh} / \mathrm{h}$ as $\rho=0.46$. It is indicate that if overtaking facilities are provided at bus stops, bus lane can be utilized more efficiently and the unnecessary dwelling time in the bus stop can be greatly reduced.

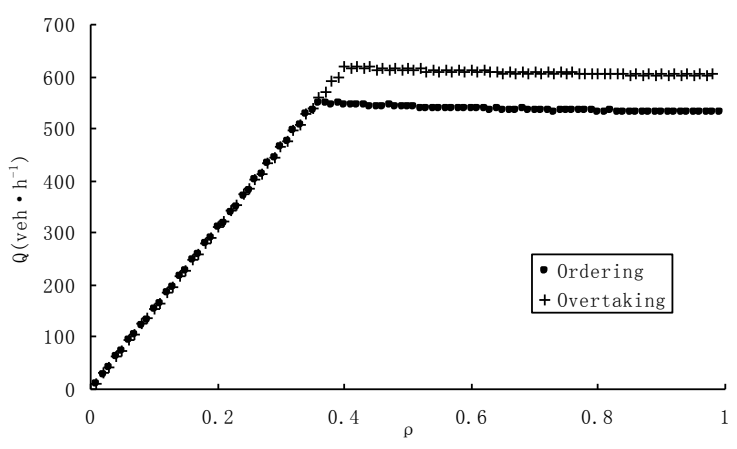

Figure. 9 The flux and density plot with different layout of off-line bus stop 


\section{SUMMARY}

In this paper, a new two-lane cellular automata model considering the influence of the bus lane on urban traffic flow under the periodic boundary condition is established. The fundamental diagrams of the traffic flow are given.

According to the analysis, the traffic of road is light and the bus ratio is small, it is not very necessary to set bus lane. If the traffic is very high or with large bus ratio, bus lane can improve the flux and velocity significantly. In order to further improve the performance and decrease the time for waiting enter or exit the bus stop, extending the bus stop length is considered. Then provision of overtaking facilities at bus stops is an especially effective way to increase flux and decrease the unnecessary dwelling time in bus stop. These implications may provide suggestions on the design of public traffic.

\section{REFERENCES}

[1] Nagel K, Schreckenberg M."A cellular automaton model for freeway traffic". J Phys.Vol. 2,pp: 2221-2229, 1992.

[2] Chowdhry D, Santen L, Schadschneider A. Statistical physics of vehicular traffic and some related systems. Phys rep, Vol.329,pp:199-329,2001.http://dx.doi.org/10.1016/S0370-1573( 99)00117-9

[3] Herbling D. "Traffic and related self-driven many-particle systems". Rev Mod Phys. Vol.73,pp:1067-1141,2001. http://dx.doi.org/10.1103/RevModPhys.73.1067

[4] P. Lin, H.L. Tan, L.J. Kong, M.R. Liu, "A study of coupling effect in cellular automata model of traffic flow for two-lane with open boundary conditions". Acta Phys . Sin .Vol.52,pp:30073012,2003.

[5] R.S. Zheng, H.L. Tan, L.J. Kong, M.R. Liu, "A cellular automaton model for mixing traffic in two-lane system".Acta Phys . Sin .Vol.54,pp:3516- 3521,2005.

[6] K.F. Wu, L.J. Kong, M.R. Liu, "The study of a cellular automaton NS and WWH mixed model for traffic flow on a two-lane roadway". Acta Phys . Sin .Vol.55,pp:6275-6280,2006.

[7] X.M. Zhao , Z.Y. Gao, B. Jia," The capacity drop caused by the combined effect of the intersection and the bus stop in a CA model". Physica A ,2007, 385 :645-658 http://dx.doi.org/10.1016/j.physa.2007.07.040
[8] X.M. Zhao , Z.Y. Gao , K. P. Li, ” The capacity of two neighbor intersections considering the influence of the bus stop". Physica A. Vol.387,pp :4649-4656,2008.

[9] Y.S. Qian, H.L. Wang, C.L. Wang, "The study of a cellular automaton traffic flow model with public transit, harbor shaped bus stop and mixed different maximum speed vehicles on single lane”. Acta Phys . Sin . Vol,57(4),pp: 2115-2120, 2008.

[10] B.Jia, X. G. Li , R. Jiang , Z .Y. Gao. "The influence of bus stop on the dynamics of traffic flow". Acta Phys. Sin . Vol.58(10).pp:6845-6851, 2009.

[11] D Q Li, L Y Dong, Dai S Q. "Investigation on traffic bottleneck induce by bus stopping with a two-lane cellular automaton model". Acta Phys . Sin . Vol.58(11)pp: 7584-7590,2009 .

[12] C.Chen, T.Wang, C.Y Li. C.W. Yuan. "Speed models of mixed traffic flow on bus-car and vehicle and analysis of traffic running state". China Journal of Highway and Transport. Vol.24,pp:97-102,126,2011.

[13] X.G Yang, H. Xu, J. Wang, K.J Long. "Study of setting conditions of bus bay stop". Chinese Journal of Theoretical and Applied Mechanics. Vol.25, pp.128-134,140,2012

[14] Y.J.Liang, Y.Xue. "The Properties of Stopping are Investigated for Off-lane Bus Station". Chinese Journal of Theoretical and Applied Mechanics. Vol.44, pp.111-116,2012

[15] S.J.Kang, Y.Xue. "Study on the Impact f Settings of Bus Stops without Bus Bay on Traffic Flow". Chinese Journal of Theoretical and Applied Mechanics. Vol.44, pp.718-726,2012.

[16] TCRP. "Transit cooperative research program report 19: guidelines for the location and design of bus stops". Transportation Research Board.1996

[17] Gardner G, Cornwell P. R, Cracknell J.A, "The performance of busway transit in developing cities(research report 329)". Transport and Road Research Laboratory.1991

\section{AUTHORS}

T. Q. Zhou is with the Sichuan University, Ph.D. student in College of Computer Science, Chengdu 610045, China. (e-mail: ztqztq@126.com).

L.J. Peng is with the Southwest University of Science and Technology, in School of Computer Science, Mianyang 621010,China. (e-mail: qiluo@126·com).

Submitted 16 September 2013. Published as re-submitted by the authors 22 October 2013. 